Supporting Information for:

\title{
Interplay of Ionic, Hydrogen-Bonding and Polar Interactions in Liquid Crystalline Complexes of a Pyridylpyridinium Polyamphiphile with (Azo)Phenol-Functionalized Molecules
}

Xavier Sallenave and C. Geraldine Bazuin*

Département de chimie, Université de Montréal,

C.P. 6128, succursale Centre-Ville, Montréal (QC), Canada H3C 3J7

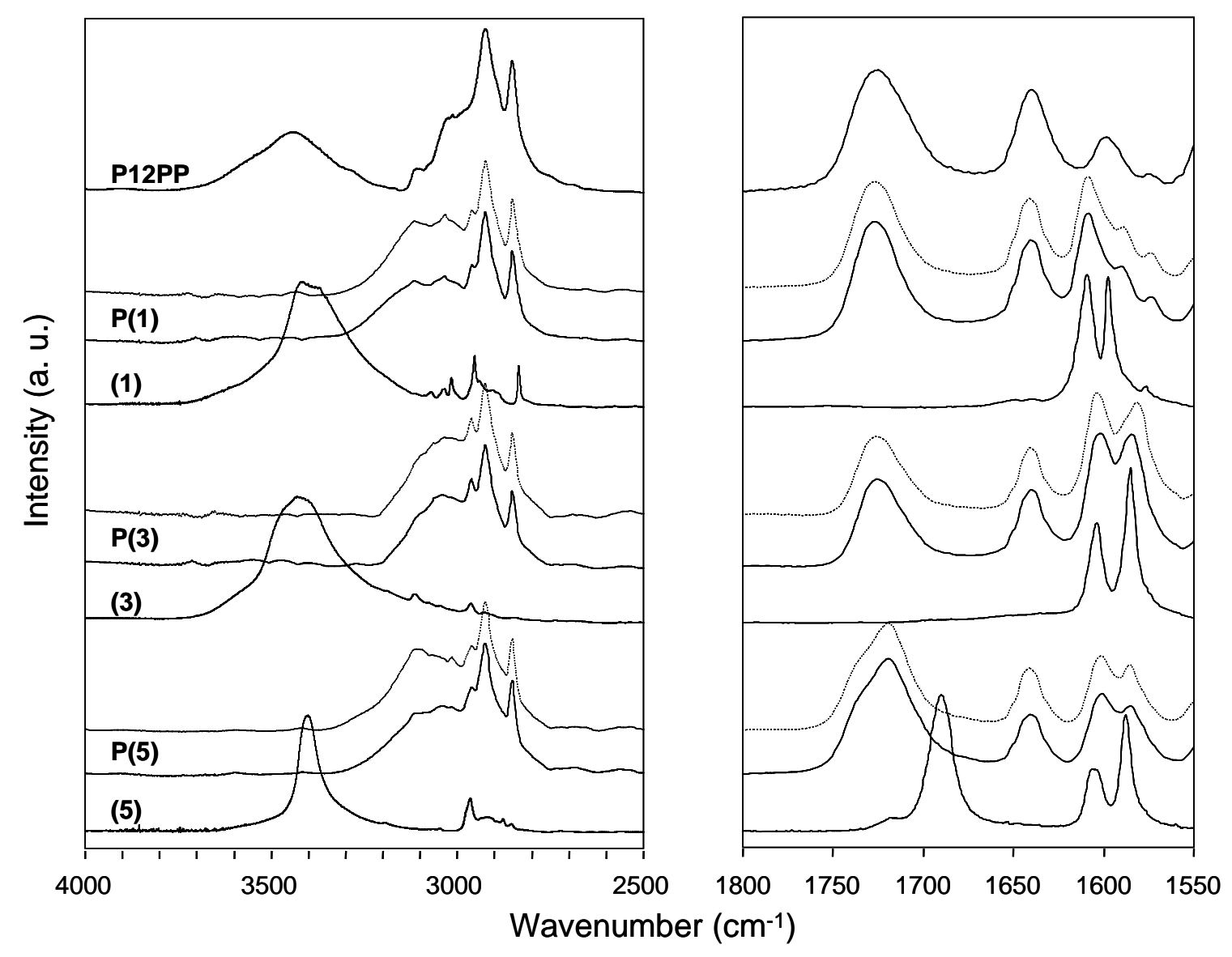

Figure S1. Ambient temperature FTIR spectra, in selected wavenumber regions, of the complexes $\mathbf{P}(\mathbf{X})$, compared with the pure components P12PP and $(\mathbf{X})$. The solid and dotted lines for $\mathbf{P}(\mathbf{X})$ give the spectra before and after melting, respectively. $\mathbf{P ( 2 )}$ and $\mathbf{P}(\mathbf{4})$ are shown in Figure S2 and in the main paper, respectively. 


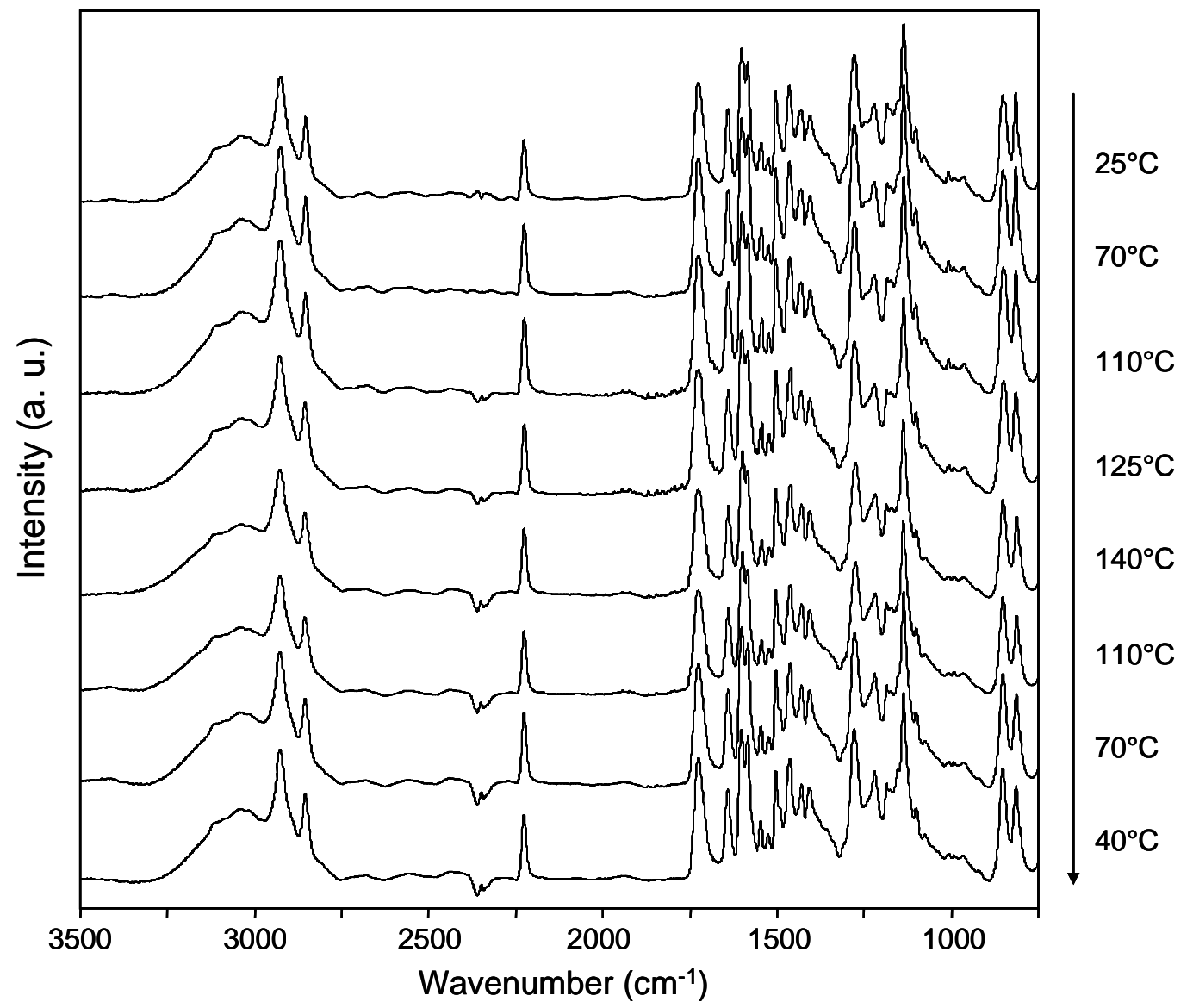

Figure S2. FTIR spectra of the complex $\mathbf{P}(2)$ taken at the temperatures given, in the order indicated by the arrow.

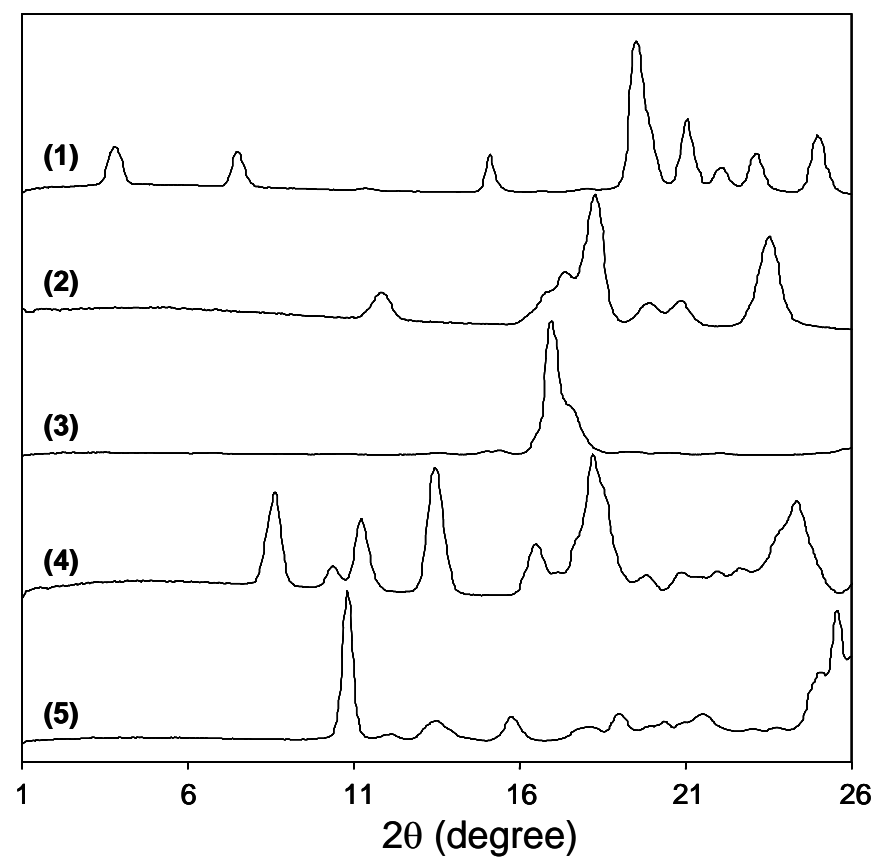

Figure S3. Ambient temperature X-ray diffractograms of the small molecules (X). 

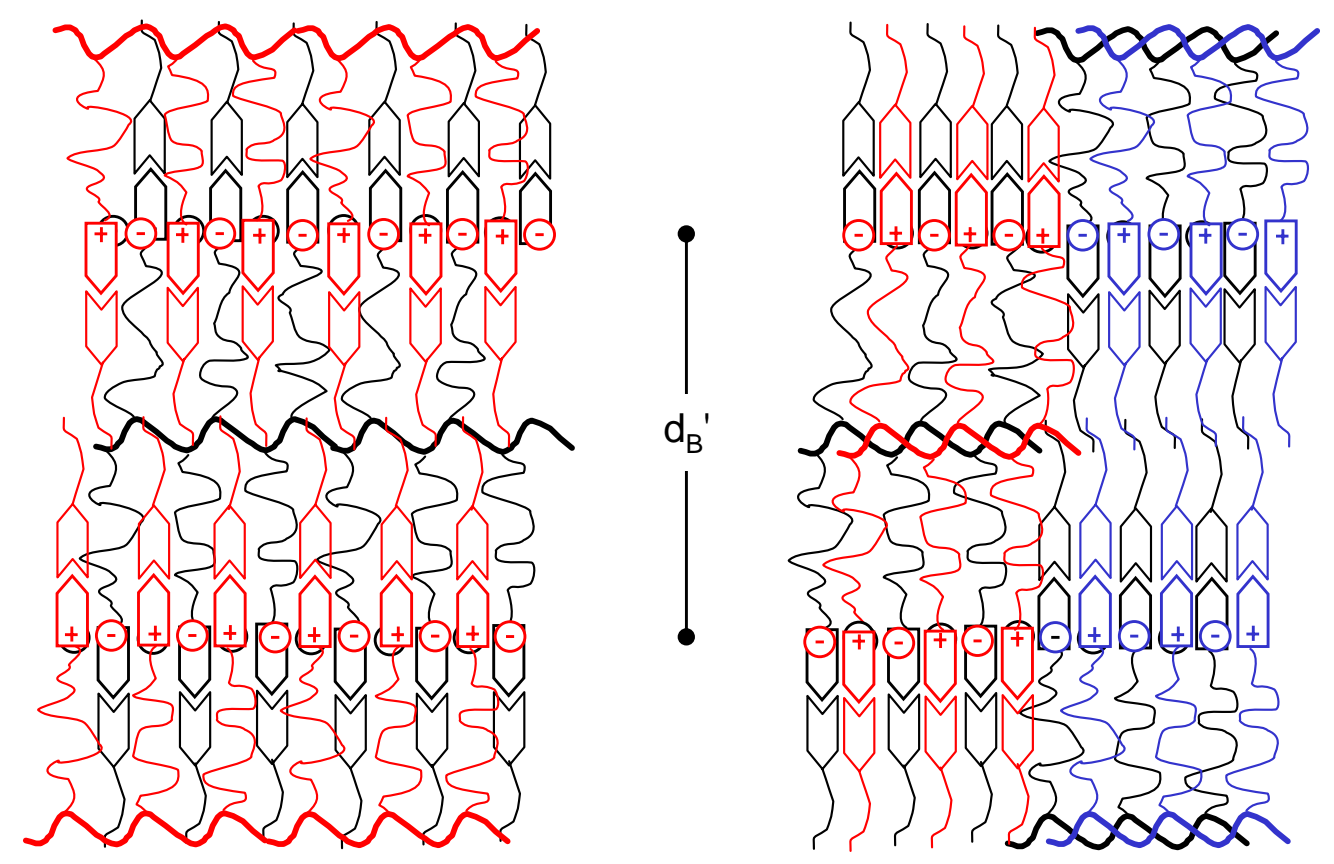

Figure S4. Three-dimensional molecular packing models illustrating possible side-chain arrangements of the $\mathbf{P ( 4 )}$ and $\mathbf{P ( 5 )}$ complexes within the SmA lamellae of thickness $d_{B}{ }^{\prime}$ [complementary to Figures 6a and 6b]. The black color can be taken to represent the motifs located in the plane ${ }^{\dagger}$ of the paper, whereas the red and blue colors represent the motifs lying above this plane. In the model on the left, the right-hand half of Figure $6 \mathrm{~b}$ is superposed on the left-hand half; this arrangement is similar to that of Figure 6a with the addition of the complexants, except that the upper and lower side-chains of Figure 6a are now located in different planes. In the model on the right, a second image of Figure $6 \mathrm{~b}$ is superposed on the first and displaced sideways by one molecular width. In both cases, the side-chains are splayed on either side of the polymer backbone, and electrostatic neutralization and space-filling are optimized. The left model seems less favorable for (tail-end) crystallization. In reality, if these packing models are indeed applicable, they may be two limiting cases for packing that may be intermediate to varying degrees throughout the material. These models would be favored by complexant tails that are miscible with the polyamphiphile spacer and sufficiently flexible for space-filling purposes.

† The word "plane" applies only for short correlation lengths, given that there is no long-range positional order within SmA lamellae. 